\title{
Artificial Neural Network based Bandwidth Estimation of a CPW-Fed Patch Antenna
}

\author{
K.Y. Kapusuz \\ Electrical and Electronics \\ Engineering Department, \\ Faculty of Engineering, Atilim \\ University, Ankara, Turkey
}

\author{
S. Can \\ Electrical and Electronics \\ Engineering Department, \\ Faculty of Engineering, Ankara \\ University, Ankara, Turkey
}

\author{
B. Dagdeviren \\ Electrical and Electronics \\ Engineering Department, \\ Faculty of Engineering, Atilim \\ University, Ankara, Turkey
}

\begin{abstract}
In this study, coplanar wave (CPW) feed microstrip antennas are designed to form dual frequency by inserting single stub. Bandwidths of the microstrip printed antennas for both upper and lower frequencies are represented in this study by considering the simulated and experimental results. For each size of T-matching network and each permittivity values are varied and effects of these variations are analyzed. The presented printed antennas in literature which have the same shape and the same feeding types are compared and results are presented. Besides presenting those, in a different way from the literature, this method is proposed to estimate the bandwidths of those antennas. The results of this study will guide researchers who want to predict the resonance frequencies and bandwidth of mentioned antennas before producing the antenna so that they will handle the problem by saving the time and estimating the bandwidth before production.
\end{abstract}

\section{General Terms}

Artificial Neural Network.

\section{Keywords}

CPW, T-matching network, printed antenna, artificial neural networks.

\section{INTRODUCTION}

Efficiency was always the most critical issue in communication systems even in first man time. Since all human need to communicate they all pay attention to increase the efficiency of the communication once in a while it was related with the smoke, once in a while it was a message bird.

In modern wireless communication systems, microstrip printed antennas are one of the most crucial components owing to their advantages. Advantages of the printed antennas can be listed as having low size, profile, weight and price [1][17]. Moreover, printed antennas can both be easily integrated to circuits [1]-[12] and provides dual and multiple frequencies via only a single antenna. However, those antennas have narrow bandwidth property, and researchers should be handled this disadvantage [1], [2], [12]. Therefore, as a way to increase the bandwidth, coplanar wave feeding (CPW) type of antennas has been used recently [1], [14], [15].

CPW not only used to increase the band, but also provides a design without any solder points on the patch [1]. Furthermore, in order to obtain dual-frequency in printed antennas different methods are used. Shorting pin-loaded, slot-loaded, slit-loaded, notch loaded and stub loaded techniques can be listed to the obtain dual and multiple frequencies. However, producing shorting pin-loaded and notch-loaded printed antennas are very challenging when feeding point and either shorting pin or notch position are close to each other.

These methods can be used to increase the bandwidth of the printed antennas. Inasmuch as upper and lower frequencies of the printed antennas sufficiently closes to each other, bandwidth of the antenna increases; however, dual-frequency antenna becomes single frequency on this occasion.

Besides, resonance frequency calculation getting more and more important, and different methods in literature are presented to calculate the resonance frequency of the printed antennas such as transmission line (TLM), cavity model and method of moment (MoM). However, obtaining closed form equation by using these methods is very hard and it takes very long time; thus, different methods or techniques such as fuzzy logic (FL), genetic algorithms (GA), and artificial neural networks (ANN) have been proposed to estimate the resonance frequency and bandwidth of the microstrip printed antennas [2]-[10]. Among them, neural network estimation has many advantages when compared with the other methods. Pros of the artificial neural network models are explained in detail by different studies in literature [18]-[24].

In recent years artificial neural network have been widely used in many applications such as, speech processing, image processing and function approximation. However, nowadays researchers are applied electromagnetic problems to both obtain accurate and save a time while solving problems.

In this study, coplanar wave single stub loaded microstrip printed antennas have been presented. Parameters of the antenna have been decided, and according to antenna parameters, lower and upper bandwidth values of the printed antenna have been estimated by using artificial neural networks model. Whereas estimating bandwidth of the printed antennas, multilayer perceptron have been used. [1] and [15] have been used while training part. More detailed information have been given in the below sections. This study is mainly conducted for estimating the bandwidth via ANN in an efficient way.

\section{ANTENNA GEOMETRY}

Geometry of the proposed printed antenna has been represented in Figure 1. 


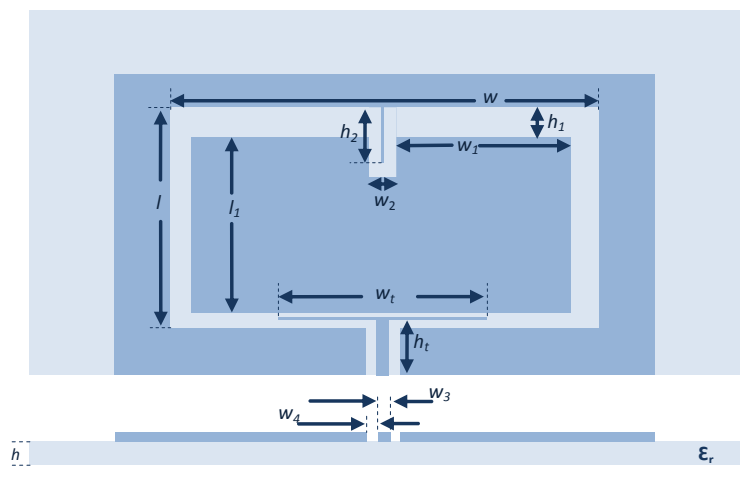

Fig 1: Geometry of antenna

$l$ and $w_{t}$ represent inner length and width of the outer frame. The distance related with the patch is illustrated with $l_{l}$ and $w_{1}$. Slot that is loaded for inserting a load an single stub width is $w_{2}$, and a height of the single stub is represented via $h_{2}$. Distance between inner and outer patch shows with $h_{l}$. In this antenna T-matching network is used to fed the antenna. The parameters of the T-matching network are $w_{t}$ and $h_{t}$ that show the width and length, respectively. Parameter of the substrate $h$ and $\varepsilon_{\mathrm{r}}$ show the thickness and permittivity, as well.

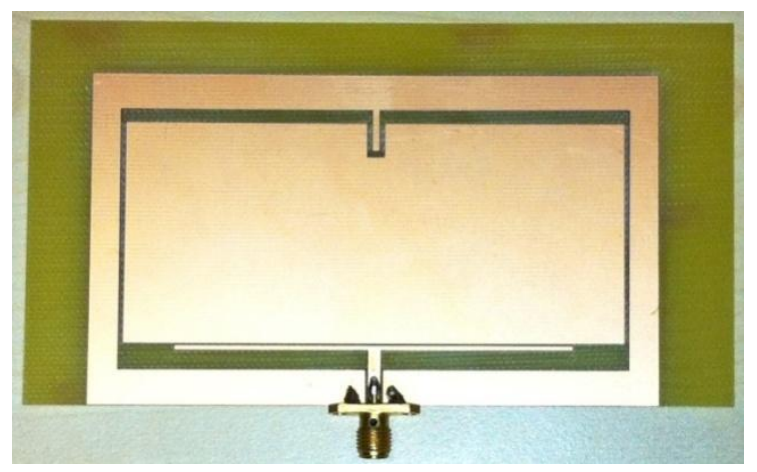

Fig 2: Produced antenna

In order to understand the accuracy of the proposed method, a prototype has been produced that is represented in Figure 2. Exact parameters of the antenna are given in Table 1. Decided antenna parameters are taken from [1]. Proposed antenna bandwidth results are given in Table 3 .

Table 1. Produced antenna parameters in $\mathbf{m m}$

\begin{tabular}{|c|c|c|c|}
\hline $\begin{array}{c}\text { Antenna } \\
\text { Parameters }\end{array}$ & $\begin{array}{c}\text { Size } \\
(\mathbf{m m})\end{array}$ & $\begin{array}{c}\text { Antenna } \\
\text { Parameters }\end{array}$ & $\begin{array}{c}\text { Size } \\
(\mathbf{m m})\end{array}$ \\
\hline$w$ & 89.8 & $l$ & 47.3 \\
\hline$w_{1}$ & 42.5 & $l_{l}$ & 40.0 \\
\hline$w_{2}$ & 3.11 & $h$ & 1.60 \\
\hline$w_{3}$ & 2.20 & $h_{1}$ & 25.0 \\
\hline$w_{4}$ & 0.46 & $h_{2}$ & 7.00 \\
\hline$w_{t}$ & 70.0 & $h_{t}$ & 10.5 \\
\hline
\end{tabular}

It should be noted that all the dimensions are given in terms of millimeters.

\section{ARTIFICIAL NEURAL NETWORKS MODEL}

The general structure of the multilayer artificial neural network is shown in Figure 3. Structure of the model has neurons and layers. All neurons in different layers are connected to each other with a weight $w_{j i}$. In training part, multilayer perceptron is selected and it consists of one input, hidden and output layers. Advantages of the multilayer perceptron can be summarize as having simple structure and achieving better accuracies when compared to the other methods in literature in most cases.

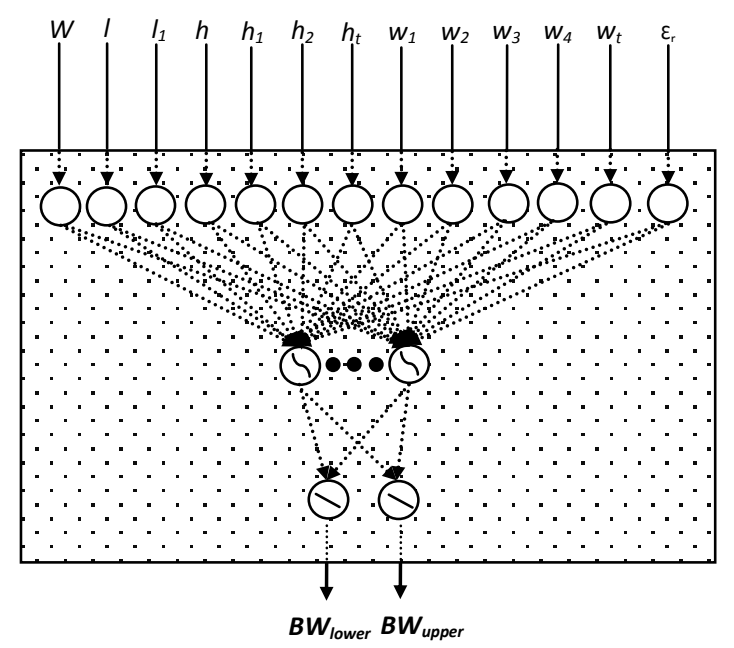

Fig 3: Structure of artificial neural networks.

In this study, the multilayer perceptron is trained by back propagation algorithm. As seen in Figure 3, the neural network is fed by the 13 antenna parameters. The bandwidths of the antenna $B W_{\text {lower }}$ and $B W_{\text {upper }}$ are considered as an output of the network. The proposed neural network model approximates the literature values of the bandwidth. The output of the hidden layer $y_{\mathrm{j}}$ is the activation function $(f)$ which is the sum of the product of input and its weight $\left(w_{\mathrm{ji}}\right)$ as formulized as Equation 1;

$$
y_{j}=f\left(\sum w_{j i} x_{i}\right)
$$

The objective of the training process is to update the synaptic weight $w_{j i}(n)$ so that the actual output of the neural network matches the desired data ones as closely as possible. The updated weights are as follows:

$w_{j i}(n)=w_{j i}(n-1)+\Delta w_{j i}(n)$

where $n$ is the iteration number and $\Delta w_{j i}(n)$ is the adjustment applied to $w_{j i}(n)$ given by

$\Delta w_{j i}(n)=\eta \delta_{j}(n) y_{i}(n)$

In Equation 2, $\eta$ and $y_{i}$ are the learning rate parameter of the algorithm and the output of the neuron $i$, as well. Moreover, $\delta$ is the local gradient in [23],[24] ; besides, output and hidden layers' local gradient can be calculated as Equation 4 and Equation 5, respectively.

$$
\begin{gathered}
\delta_{j}=\left(\frac{\partial f}{\partial n e t_{j}}\right)\left(y_{j}{ }^{(t)}-y_{j}\right) \\
\delta_{j}=\left(\frac{\partial f}{\partial n e t_{j}}\right) \sum_{q} w_{q j} \delta_{q}
\end{gathered}
$$

\subsection{Training process}

Antenna parameters are demonstrated in Table 2 which are used to train artificial neural networks structure. Indeed all 
data in table are taken from literature in [1], and the antenna parameters except wt and permittivity are the same as the produced one. The bandwidths of these antennas are examined according to permittivity and $w_{t}$. It is a well known fact that all antenna parameters have an effect on the bandwidth of the microstrip printed antenna. However, in order to reduce the computational burden, some parameters can be taken into account as a constant in the neural networks approach.

The proposed networks training set values are all taken from the available literature and they are presented in Table 2. When the network is fully trained, the neural network computes the approximated bandwidths with a significant low error as its output. These outputs can be expressed as a function of its inputs and corresponding desired outputs'. The average error between the values resulting from the desired and the actual values are a measure of how well the network meets the bandwidth values. Therefore, this difference is used as a performance criterion for the proposed method. What is more learning rate and epochs are assigned as 0.4 and 2000, respectively.

Table 2. Bandwidth values in [1]

\begin{tabular}{|c|c|c|c|c|}
\hline \multirow[b]{2}{*}{ Antenna } & \multirow[b]{2}{*}{$w_{t}$} & \multirow[b]{2}{*}{$\varepsilon_{r}$} & \multicolumn{2}{|c|}{ Desired Value } \\
\hline & & & $\begin{array}{c}B W_{\text {lower }} \\
\text { (MHz) }\end{array}$ & $\begin{array}{c}\text { BW } \\
\text { (MHper }\end{array}$ \\
\hline 1 & 60 & 2.2 & 231 & 511 \\
\hline 2 & 50 & 2.2 & 156 & 531 \\
\hline 3 & 70 & 2.94 & 416 & 470 \\
\hline 4 & 70 & 3 & 411 & 403 \\
\hline 5 & 60 & 3 & 321 & 341 \\
\hline 6 & 50 & 3 & 276 & 406 \\
\hline 7 & 60 & 4.4 & 321 & 410 \\
\hline 8 & 50 & 4.4 & 295 & 491 \\
\hline 9 & 40 & 4.4 & 165 & 612 \\
\hline 10 & 70 & 6.15 & 316 & 306 \\
\hline 11 & 70 & 9.18 & 215 & 185 \\
\hline 12 & 50 & 9.18 & 135 & 241 \\
\hline
\end{tabular}

\section{RESULTS}

Results of the proposed network structure are presented in Table 3. The proposed results in this table for $1^{\text {st }}, 2^{\text {nd }}$ and $3^{\text {rd }}$ antennas are obtained via finite element method based simulation program.

Table 3. Comparison between desired and proposed bandwidth values

\begin{tabular}{|c|c|c|c|c|c|c|c|c|}
\hline \multirow{2}{*}{ Antenna } & \multirow{2}{*}{$\varepsilon_{r}$} & \multirow{2}{*}{$\boldsymbol{w}_{\boldsymbol{t}}$} & \multicolumn{3}{|c|}{ Bandwidth (MHz) } & \multicolumn{2}{c|}{$\begin{array}{c}\text { Desired } \\
\text { Value }\end{array}$} & \multicolumn{2}{|c|}{ ANN } & \multicolumn{2}{c|}{ Error(\%) } \\
\cline { 5 - 9 } & & & low & up & low & up & low & up \\
\hline 1 & 2.2 & 70 & 421 & 436 & 418 & 440 & 0.71 & 0.91 \\
\hline 2 & 4.4 & 70 & 376 & 396 & 370 & 401 & 1.06 & 1.26 \\
\hline 3 & 9.18 & 60 & 195 & 236 & 192 & 235 & 1.54 & 0.42 \\
\hline [Produced] & 4.4 & 70 & 345 & 375 & 337 & 382 & 2.32 & 1.87 \\
\hline \multicolumn{10}{c|}{ Average Error (\%) } \\
\hline
\end{tabular}

According to changes both dielectric constant and $\mathrm{w}_{\mathrm{t}}$ values are represented in Table 3. To test the proposed model different dielectric constant and $\mathrm{w}_{\mathrm{t}}$ values have been chosen.
For both variations, dielectric constant and $\mathrm{w}_{\mathrm{t}}$, proposed model, ANN, gives significant agreements. For the first antenna, that has a permittivity value of 2.2 and $\mathrm{w}_{\mathrm{t}}$ value of $70 \mathrm{~mm}$. Desired values and proposed ANN model has an error of $0.71 \%$ for the lower and an error of $91 \%$ for the upper. To validate the accuracy of the proposed ANN model an antenna produced and the results are shown in antenna picture. When ANN model is compared with the desired value, lower and upper bandwidths have $2.32 \%$ and $1.87 \%$ error, respectively. Moreover, average error of the ANN model is $1.41 \%$ for lower bandwidth and $1.12 \%$ for upper bandwidth.

Besides, although both antenna 2 and produced one has the same antenna parameters, they have errors for $8.99 \%$ of lower bandwidth and $5.6 \%$ of upper bandwidth. On the other hand, proposed ANN model bandwidth estimator gives better results when compared with the finite element method based simulation program since errors are smaller between produced antenna and ANN model as shown in Table 2.

\section{CONCLUSION}

In this study, a demand on estimating the bandwidth of the CPW-fed microstrip patch antenna is considered and in order to estimate the bandwidths of the proposed printed antennas, new model have been suggested. Difficulty in determining an expression for the bandwidth with a closed form expression lead the authors of this paper to use ANN abilities by which both accuracy and time are gained. This model reduces the computation time since not any calculation is required to determine the bandwidth of the $\mathrm{CPW}$-fed microstrip patch antennas. It also increases the accuracy of the estimation of the bandwidth for $1.41 \%$ for the lower and $1.12 \%$ for the upper frequencies. The results of this study will be the basement of the latter studies that predict the resonance frequencies and the bandwidth of mentioned antennas before producing the antenna so that the engineers will handle the problem by saving the time and estimating the frequency and bandwidth before production.

\section{REFERENCES}

[1] Dagdeviren, B., Kapusuz, K.Y., Can, S. and Aydin, E. 2013. Analysis of the parameters that effects the operating frequencies and bandwidth of a cpw-fed patch antenna. IEEE 21th Signal Processing and Communications Applications Conference (SIU).

[2] Dagdeviren, B., Kapusuz, K.Y., Can, S. and Aydin, E. 2013. Design and fabrication of dual-frequency h-shaped antenna. IEEE 21th Signal Processing and Communications Applications Conference (SIU).

[3] E. Aydin, S. Can, "Modified resonant frequency computation for tunable equilateral triangular microstrip patch," IEICE Electronics Express, 2010, vol. 7, no. 7, pp. 500-505.

[4] S. Can, Computation of Resonant Frequency of Dual Band Triangular Patch Antenna, Master's Thesis in Electrical \& Electronics Engineering, Atilim University, Turkey, July 2011.

[5] E. Aydin, S. Can, "Operating frequency calculation of a shorting pin loaded ETMA," Microwave and Optical Technology Letters, 2012, vol. 54, no. 6, 1 pp.432-1435.

[6] Can, S., Kapusuz, K.Y. and Aydin, E. 2012. Dualfrequency, two shorting pin loaded equilateral triangular patch antennas. 32th Progress In Electromagnetics Research Symposium (PIERS). 
[7] Can, S., Kapusuz, K.Y. and Aydin, E. 2012. Analysis of a dual frequency circular patch antenna. 32th Progress In Electromagnetics Research Symposium (PIERS).

[8] Can, S., Kapusuz, K.Y. and Aydin, E. 2012. Dualfrequency, single shorting pin loaded equilateral triangular patch antennas. 12th Mediterranean Microwave Symposium (MMS).

[9] Can, S., Kapusuz, K.Y. and Aydin, E. 2012. Design \& analyzes of dual-frequency circular microstrip patch antenna with a shorting pin. 12th Mediterranean Microwave Symposium (MMS).

[10] Kapusuz, K.Y., Can, S. and Aydin, E. 2012. Rectangular shaped, dual-frequency antenna with a single shorting pin. 9th International Conference on Electronics, Computer and Computation (ICECCO).

[11] Gao, S. C., Li, L. W., Yeo, T. S., Leong, M. S. 2001. A dual-frequency small microstrip antenna. International Symposium on Antennas and Propagation Society.

[12] Kaynakoz, G., Kilic, N. K., Kapusuz, K.Y., Can, S. and Aydin, E. 2012. Reader antenna design for RFID applications. IEEE 21th Signal Processing and Communications Applications Conference (SIU).

[13] J.S. Row, K.W. Lin, "Low-profile design of dual frequency and dual polarized triangular microstrip antennas," Electronics Letters, 2004, vol. 40, no. 3, pp. 156-157.

[14] Y.C. Chen, S. Y. Chen, P. Hsu, "A modified cpw-fed slot loop antenna with reduced cross polarization and size," Antennas and Wireless Propagation Letters, 2011, vol. 10, pp. 1124-1126.

[15] D. Llorens, P. Otero, C. C. Penalosa, "Dual-band, single CPW port, planar slot antenna," Transactions on Antennas and Propagation, 2003, vol. 51, no. 1, pp. 137139.

[16] Y. C. Lin, K. J. Hung, "Design of dual-band slot antenna with double T-match stubs," Electronics Letters, 2006, vol. 42 , no. 8 , pp. 438-439.
[17] S. Maci, G. B. Gentili, "Dual frequency patch antennas," Antennas and Propagation Magazine, 1997, vol. 39, no. 6, pp. 13-20.

[18] Marzall, L.F. and Lacava, J. C. S. 2010. Bandwidth estimating strategy for a 2-layer rectangular suspended microstrip antenna. 29th Progress In Electromagnetics Research Symposium (PIERS).

[19] Uzer, D., Uzer, M.S., Gultekin, S. S. and Yilmaz, N. 2012. Effect of U-slot applications on circular microstrip patches modeling with artificial neural networks on impedance bandwidth. 30th Progress In Electromagnetics Research Symposium (PIERS).

[20] Uzer, M. S., Uzer, D., Yilmaz, N. and Gultekin, S. S. 2012. Bandwidth modeling of U-slot rectangular microstrip antennas with artifical neural networks. 30th Progress In Electromagnetics Research Symposium (PIERS).

[21] Rao, N. and Kumar, D. V. 2011. Gain and bandwidth enhancement of microstrip antenna using partial substrate removal in multiple-layer dielectric substrate. 28th Progress In Electromagnetics Research Symposium (PIERS).

[22] Fan, Z. and Mars, P. 1996. Application of artificial neural networks to effective bandwidth estimation in ATM networks. IEEE International Conference on Neural Networks.

[23] Haykin, S.; Neural Networks-A Comprehensive Foundation, Macmillan College Publishing Company, 1994.

[24] K. Y. Kapusuz, H. Tora, S. Can, "Neural network based estimation of resonant frequency of an equilateral triangular microstrip patch antenna," Technical Gazette, in press. 